\title{
Theoretical Investigation on Electronic and Magnetic Structures of FeRh
}

\author{
Hidekazu Takahashi, Masaaki Araidai*, Susumu Okada and Kenji Shiraishi* \\ Graduate School of Pure and Applied Sciences,University of Tsukuba, 1-1-1 Tennodai, Tsukuba, Ibaraki 305-8571, Japan \\ "Institute of Materials and Systems for Sustainability, Furo-cho, Chikusa-ku, Nagoya, 464-8603, Japan
}

In order to clarify the mechanism behind antiferromagnetic (AFM)-ferromagnetic (FM) phase transition, we investigate the electronic and magnetic structures of FeRh by using first principles calculations with the GGA $+U$ method. By choosing the appropriate values of the on-site Coulomb interaction ( $U$ ) of $\mathrm{Fe}_{3 \mathrm{~d}}$ and $\mathrm{Rh}_{4 \mathrm{~d}}$ electrons, we succeed in explaining the reported AFM-FM phase transition experiments for the first time by obtaining the total energy difference between the AFM and FM states $(\Delta E)$. Other physical quantities such as the density of states (DOS) are also consistent with experimental reports.

Key words: magnetic refrigeration, inverse magnetocaloric material, antiferromagnetic-ferromagnetic phase transition, GGA $+U$ method, on-site Coulomb interaction

\section{Introduction}

Room-temperature magnetic refrigeration technology using magnetocaloric materials ( $\mathrm{MCMs}$ ) has recently gained attention because it is expected to eliminate refrigerants that are greenhouse gases and improve the system efficiency of refrigerators. ${ }^{1)}$ Various performance-enhancing MCMs such as $\mathrm{La}\left(\mathrm{Fe}_{\mathrm{x}} \mathrm{Si}_{1-\mathrm{x}}\right)_{13} \mathrm{H}_{\mathrm{y}},{ }^{2)} \mathrm{MnFe}(\mathrm{P}, \mathrm{Si}),{ }^{3)}$ and so on have been found so far. Inverse magnetocaloric materials (IMCMs) such as $\mathrm{FeRh}^{4}$ ) and $\mathrm{Mn}_{2 \times \mathrm{x}} \mathrm{Cr}_{\mathrm{x}} \mathrm{Sb}^{5)}$ have also been the focus of attention because of the appearance of the 'giant' inverse magnetocaloric effect (IMCE) under a low magnetic field. In particular, FeRh shows a first-order antiferromagnetic (AFM)-ferromagnetic (FM) phase transition at $T_{\operatorname{tr}} \approx 320 \sim 370 \mathrm{~K}$ without a magnetic field and exhibits a giant adiabatic temperature change $\Delta T_{\mathrm{ad}}$ (= $13 \mathrm{~K})$ under $1.95 \mathrm{~T}$. Recent studies suggest that $\Delta T_{\mathrm{ad}}$ can be increased up to $18 \mathrm{~K}$ per $1 \mathrm{~T}$. ${ }^{6)}$ The appearance of new MCMs and IMCMs that are superior to FeRh is now expected. It is quite important to understand the why $\Delta T_{\text {ad }}$ is large in FeRh. Moreover, it is essential to clarify the microscopic mechanism behind the AFM-FM phase transition in order to develop new magnetocaloric materials.

Since FeRh was discovered by Fallot in 1938,7) various experiments ${ }^{8)-19)}$ on its physical parameters have been performed, such as the difference between the minimum total energy of the FM state and that of the AFM state $(\Delta E)$ related to $T$ tr. ${ }^{10)}$

Various theoretical investigations have also been carried out. For example, Kittel proposed the exchange inversion model. ${ }^{20}$ ) There have also been various theories proposed. However, all of these currently remain the subject of debate. Several investigations into electronic and magnetic structures using various band-structure calculation methods have been reported to date. ${ }^{21)-28)} \mathrm{We}$ should point out here that recent advanced first-principles calculations ${ }^{26)-28)}$ are not able to reproduce important physical quantities such as $\Delta E$. In particular, if $\Delta E$ calculations are inconsistent with experiments, its mechanism as an IMCM cannot be clarified. Generally, in alloys containing $3 \mathrm{~d}$ and $4 \mathrm{~d}$ transition metals, regard for electronic correlation is known to be important. However, this is not considered in all of the reported calculations, including Refs. (26)-(28). Therefore, we treat this electronic correlation as the on-site Coulomb interaction $(U)$ and examine the effect of $U$ on various physical quantities, particularly $\Delta E$. In this paper, we investigate the electronic and magnetic structures of FeRh and have succeeded in reproducing the AFM-FM phase transition quantitatively for the first time.

\section{Calculation Method}

In $\mathrm{FeRh}$, which is a $\mathrm{CsCl}$ crystal with a simple cubic structure, the magnetic structures in the AFM and FM states are shown in Fig. 1. In order to treat FeRh in both the AFM and FM states, the crystal is considered a face-centered cubic structure with lattice constant $a=2 a$, where $a$ is the lattice constant in the case of a simple cubic structure. The atomic positions of two Fe atoms are $(0.0,0.0,0.0)$ and $\left(0.5 a^{\prime}, 0.5 a^{\prime}, 0.5 a^{\prime}\right)$. The atomic positions of two $\mathrm{Rh}$ atoms are (0.25 a', $\left.0.25 \mathrm{a}^{\prime}, 0.25 \mathrm{a}^{\prime}\right)$ and (0.75 a', $\left.0.75 a^{\prime}, 0.75 a^{\prime}\right)$.

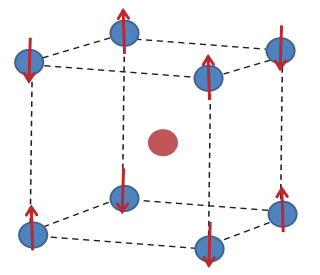

(a)

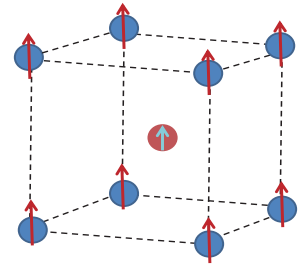

(b)
Fig. 1 Magnetic structure of (a) AFM and (b) FM states of FeRh. The blue and violet circles indicate $\mathrm{Fe}$ and $\mathrm{Rh}$ atoms, respectively. $\uparrow$ and $\downarrow$ indicate the directions of the magnetic moments.

The Vienna ab initio simulation package (VASP) 29),30) with the projector augmented wave (PAW) method ${ }^{31,32)}$ was utilized for performing the first principles 
calculations. We used the Perdew-Burke-Ernzerhof exchange-correlation functional ${ }^{33}$ ) with the Hubbard $U$ method (GGA $+U)^{34)}$ including $U$ and the exchange parameter $(J)$ for each atomic orbital in the materials, as our GGA calculation results such as $\Delta E(=31.5$ meV/atom) and the magnitude relation of $\mathrm{Fe}$ magnetic moment values between the AFM and FM states $(3.12 \mu \mathrm{B}$, $\left.3.18 \mu_{\mathrm{B}}\right)$ were inconsistent with experimental results. In the calculations, the Dudarev approach, which is only dependent on $U_{\text {eff }}=U-\mathcal{J}^{\beta 5}$ ), was adopted. The magnitude of $U$ and $J$ for the Fesd electron $\left(U_{\mathrm{Fe}}, J_{\mathrm{Fe}}\right.$ ) and for the $\mathrm{Rh}_{4 \mathrm{~d}}$ electron $\left(U_{\mathrm{Rh}}, J_{\mathrm{Rh}}\right)$ were set to $(2.0 \mathrm{eV}, 1.0$ $\mathrm{eV})$ and $(1.95 \mathrm{eV}, 1.0 \mathrm{eV})$, respectively so that the obtained physical quantities of the Fe and Rh crystals (lattice constant, bulk modulus and density of states (DOS)) using these $U$ and $J$ were consistent with the reported experiments. The Methfessel-Paxton method ${ }^{36)}$ was adopted by choosing the width of the smearing $\Delta \sigma=$ $0.05 \mathrm{eV}$. A plane-wave basis set with an energy cutoff $\left(E_{\text {cut }}\right)$ of $830 \mathrm{eV}$ and an $11 \times 11 \times 11$ Monkhorst-Pack k points mesh ${ }^{37)}$ was used. ${ }^{38)}$

\section{Results and Discussion}

A comparison of the physical quantities obtained in the present analysis by using the above GGA $+U$ calculation method and those reported in experiments is given in Table I. The calculation results are detailed as follows. First, the relationship between the total energies of the AFM and FM states and the lattice constant is investigated by choosing appropriate magnitudes of $U_{\mathrm{Fe}}$ and $U_{\mathrm{Rh}}$ to estimate $\Delta E$. The obtained results are shown in Fig. 2. As shown, the AFM state is stable when the lattice constant is small. With increasing the lattice constant, the total energy of the AFM state has a minimum at lattice constant (aAFM) (= $2.99 \AA$ ) and the total energy difference between the AFM and FM states becomes smaller. When the lattice constant is increased further, the total energy of the FM state has a minimum at lattice constant (aFM) (= $3.01 \AA)$. Finally, the FM state becomes stable. The obtained values for aAFM and aFM are consistent with the experiment $\left(a_{\mathrm{AFM}}=2.981 \AA \text { and } \mathrm{aFM}=2.999 \AA\right)^{9}{ }^{9}$ It is noted that the obtained value for $\Delta E(=2.71 \mathrm{meV} / \mathrm{atom})$

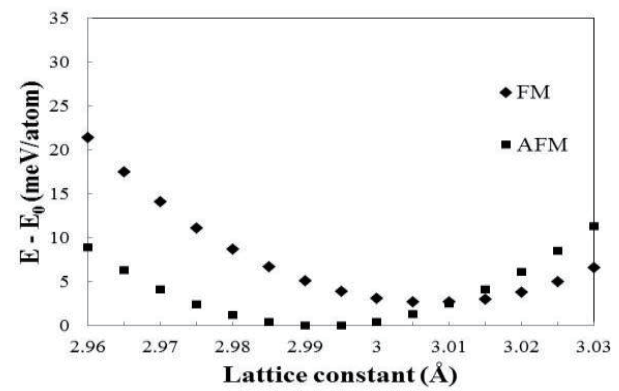

Fig. 2 Total energy versus lattice constant. On the basis of the minimum of the AFM state, the closed squares and the diamonds indicate the total energies of the AFM and the FM states, respectively. is consistent with the experimental value $\Delta E(=2.80$ meV/atom). ${ }^{10)}$

The other physical quantities that were obtained are detailed as follows. The magnetic moments of the Fe and $\mathrm{Rh}$ atoms $\left(m_{\mathrm{Fe}}, m_{\mathrm{Rh}}\right)$ in the AFM and FM states are investigated. The obtained magnetic moments are almost identical to those in the experiments. ${ }^{11)}$ The obtained $m_{\mathrm{Fe}}$ of the AFM state $\left(=3.31 \mu_{\mathrm{B}}\right)$ becomes equal to $m_{\mathrm{Fe}}$ of the $\mathrm{FM}$ state $\left(=3.31 \mu_{\mathrm{B}}\right)$, which is slightly different from the tendency obtained by the experiment $\left(m_{\mathrm{Fe}}\right.$ of the AFM state $>m_{\mathrm{Fe}}$ of the FM state). In all of the reported calculations ${ }^{21-28)}$, the relationship whereby $m_{\mathrm{Fe}}$ of the AFM state $<m_{\mathrm{Fe}}$ of the FM state is satisfied. However, in the present work, the magnitude relation of the obtained $m_{\mathrm{Fe}}$ values between the AFM and FM states is approaching that of the experiment.

The obtained relationship between the obtained values of $m \mathrm{Fe}$ and $m_{\mathrm{Rh}}$ of the AFM and FM states and the lattice constant is shown in Fig. 3. With increasing the lattice constant, (i) the change in $m_{\mathrm{Fe}}$ is much larger than that in $m_{\mathrm{Rh}}$, (ii) the change in $m_{\mathrm{Fe}}$ in the AFM state is larger than that in the FM state, and (iii) the magnitude relation of $m_{\mathrm{Fe}}$ values between the AFM and FM states is reversed near the observed lattice constant.

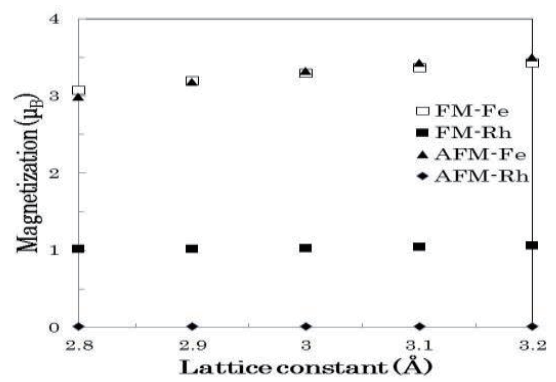

Fig. $3 m_{\mathrm{Fe}}$ and $m_{\mathrm{Rh}}$ of the AFM and FM states versus lattice constant.

The critical magnetic field at $0 \mathrm{~K}\left(H_{\mathrm{c}}(0)\right)$ which can provide us with useful information to understand the IMCE of FeRh can be obtained by ${ }^{22)}$

$$
H_{\mathrm{c}}(0)=\Delta E /\left(M_{\mathrm{FM}}-M_{\mathrm{AFM}}\right),
$$

where $M_{\mathrm{FM}}$ and $M_{\mathrm{AFM}}\left(=0 \mu_{\mathrm{B}}\right)$ are the magnetization per atom of the FM and AFM states, respectively. The obtained $H_{\mathrm{c}}(0) \quad(=21.6 \mathrm{~T})$ is consistent with the experiments $(=21.2 \mathrm{~T} \sim 29.7 \mathrm{~T}){ }^{12), 13), 14)}$

The DOS and the partial density of states (PDOS) near the Fermi level $\left(E_{\mathrm{F}}\right)$ of the Fe and Rh atoms of the FM and AFM states were investigated and are shown in Figs. 4(a)-(d) and Figs. 5(a)-(d), respectively. The distribution of DOS near $E_{\mathrm{F}}$ of the FM state shown in Figs. 4(a) and (b) and of the AFM state shown in Figs. 4(c) and (d) are similar to the reported photoemission spectra. ${ }^{15), 16)}$ In particular, the main peak positions of DOS near $E_{\mathrm{F}}$ are around $-5.0 \mathrm{eV}$ to $-4.0 \mathrm{eV}$ and around $-3.0 \mathrm{eV}$ to $-2.0 \mathrm{eV}$. Moreover, the obtained ratio of DOS at $E_{\mathrm{F}}$ of the FM state and that of the AFM state $\left(D\left(E_{\mathrm{F}}\right)_{\mathrm{FM}} / D\left(E_{\mathrm{F}}\right)_{\mathrm{AFM}}\right)$ (= $4.72)$ is also consistent with the experiments. ${ }^{17), 18)}$ The reason the obtained value of $D\left(E_{\mathrm{F}}\right)_{\mathrm{FM}} / D\left(E_{\mathrm{F}}\right)_{\mathrm{AFM}}$ is large 
is examined by using PDOSs, as shown in Figs. 5(a)-(d), respectively. PDOS at $E_{\mathrm{F}}\left(P D\left(E_{\mathrm{F}}\right)\right)$ of a $\mathrm{Fe}$ atom of the FM state for the minority spin state is much higher than that of the AFM state for the minority spin state, while $P D\left(E_{\mathrm{F}}\right)$ of an Fe atom for both the AFM and FM states for the majority spin state is low and $P D\left(E_{\mathrm{F}}\right)$ of an $\mathrm{Rh}$ atom of both the AFM and FM states for the majority spin and minority spin state are also low. Therefore, $D\left(E_{\mathrm{F}}\right)_{\mathrm{FM}} / D\left(E_{\mathrm{F}}\right)_{\mathrm{AFM}}$ becomes large due to the large change in the contribution of Fesd electrons to $D\left(E_{\mathrm{F}}\right)$ in the AFM-FM phase transition.

The effect of $U$ on $\Delta E$ and $m_{\mathrm{Fe}}$ in the AFM and FM states is investigated using PDOS. Comparing Fig. 5(b) with Fig. 5(d), magnetic states of Rh for the AFM and FM states are nonmagnetic and FM states, respectively. When the magnitude of $U_{\mathrm{Rh}}$ is increased to $1.95 \mathrm{eV}$, the
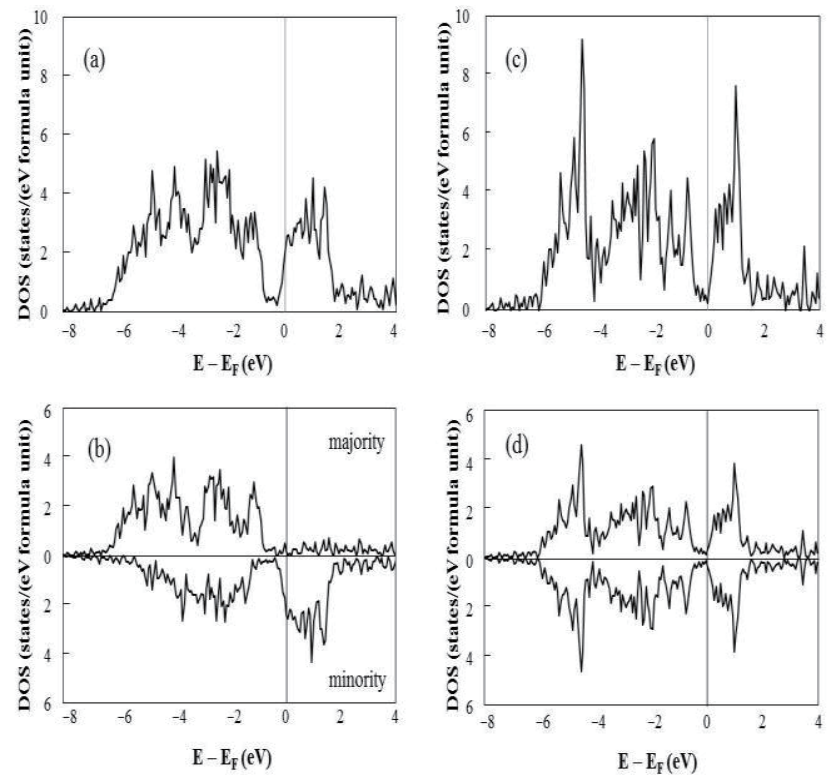

Fig. 4 DOS near $E_{\mathrm{F}}$ of (a) the FM state, (b) the FM state for both the majority spin and the minority spin states, (c) the AFM state, and (d) the AFM state for each of the majority spin and the minority spin states. nonmagnetic state becomes unstable and the FM state is energetically advantageous. As a result, $\Delta E$ becomes smaller compared with the GGA calculation result. From Fig. 5(a) and (c), we can see that the Fesd band in the majority spin state for the AFM and FM states is almost filled, while that in the minority spin state for the AFM and FM states is inadequately filled. Similar results (not shown) were obtained by the GGA calculation. When the magnitude of $U_{\mathrm{Fe}}$ is increased to $2.0 \mathrm{eV}, \mathrm{Fe}_{3 \mathrm{~d}}$ band at $E_{\mathrm{F}}$ in the minority spin state moves to the upper energy side, which results in a decrease of the occupied $\mathrm{Fe}_{3 \mathrm{~d}}$ PDOS in the minority spin state. This leads to an increase of $m \mathrm{Fe}$ in the $\mathrm{AFM}$ and $\mathrm{FM}$ states and improvement of the magnitude relation of $m_{\mathrm{Fe}}$ values between the AFM and FM states. From the above results, the effect of $U$ plays a crucial role in discussing the AFM-FM phase transition.
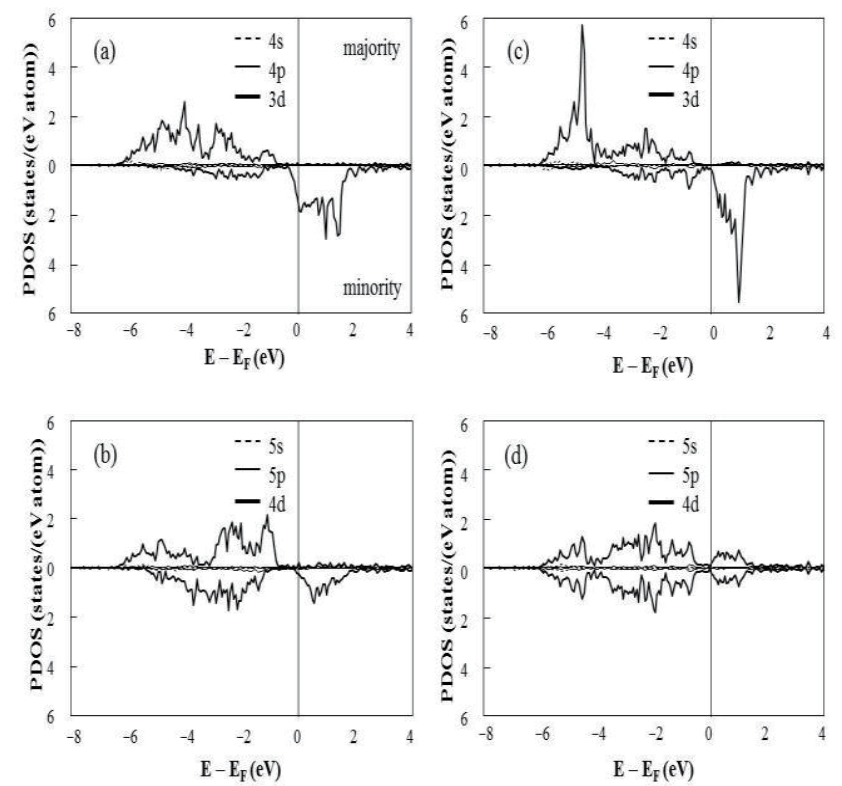

Fig. 5 PDOS near $E$ F of (a) an Fe atom of the FM state, (b) an Rh atom of the FM state, (c) an Fe atom of the AFM state and (d) an Rh atom of the AFM state.

Table I Comparison of physical quantities obtained in the present analysis using the GGA $+U$ method and those reported in experiments.

\begin{tabular}{|c|c|c|c|c|c|c|c|c|c|c|c|c|}
\hline & \multirow{2}{*}{$\begin{array}{c}\mathrm{AFM} \\
a_{\mathrm{AFM}} \\
\AA \\
\AA\end{array}$} & \multirow{2}{*}{$\begin{array}{c}\mathrm{FM} \\
a \mathrm{FM} \\
\AA \\
\end{array}$} & \multirow[b]{2}{*}{$\begin{array}{l}\Delta E \\
\mathrm{meV}\end{array}$} & \multicolumn{2}{|c|}{$\overline{\mathrm{AFFM}}$} & \multicolumn{2}{|c|}{ 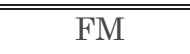 } & \multirow[b]{2}{*}{$\begin{array}{c}H_{\mathrm{c}}(0) \\
\mathrm{T}\end{array}$} & \multirow[b]{2}{*}{$\begin{array}{l}D\left(E_{\mathrm{F}}\right)_{\mathrm{FM}} / \\
D\left(E_{\mathrm{F}}\right)_{\mathrm{AFM}}\end{array}$} & \multirow[b]{2}{*}{$\begin{array}{c}\text { YFM/ } \\
\text { YAFM }^{\text {i) }}\end{array}$} & \multirow{2}{*}{$\begin{array}{c}\text { AFM } \\
B_{\mathrm{AFM}^{\mathrm{ii}}} \\
\mathrm{GPa} \\
\end{array}$} & \multirow{2}{*}{$\begin{array}{c}\mathrm{FM} \\
B_{\mathrm{FM}^{\mathrm{iii}}} \\
\mathrm{GPa} \\
\end{array}$} \\
\hline & & & & $\begin{array}{c}m_{\mathrm{Fe}} \\
\mu_{\mathrm{B}}\end{array}$ & $\begin{array}{c}m_{\mathrm{Rh}} \\
\mu_{\mathrm{B}}\end{array}$ & $\begin{array}{c}m_{\mathrm{Fe}} \\
\mu_{\mathrm{B}}\end{array}$ & $\begin{array}{c}m_{\mathrm{Rh}} \\
\mu_{\mathrm{B}}\end{array}$ & & & & & \\
\hline $\begin{array}{l}\text { present } \\
\text { work }\end{array}$ & 2.99 & 3.01 & 2.71 & 3.31 & 0 & 3.31 & 1.04 & 21.6 & 4.72 & & 195.7 & 210.6 \\
\hline Ref. (8) & 2.987 & 2.997 & & & & & & & & & & \\
\hline Ref. (9) & 2.981 & 2.999 & & & & & & & & & & \\
\hline Ref. (10) & & & 2.80 & & & & & & & 2.41 & $142 \pm 14$ & $133 \pm 20$ \\
\hline Ref. (11) & & & & 3.30 & 0 & 3.17 & 0.97 & & & & & \\
\hline Ref. (12) & & & & & & & & 23.4 & & & & \\
\hline Ref. (13) & & & & & & & & 29.7 & & & & \\
\hline Ref. (14) & & & & & & & & 21.2 & & & & \\
\hline Ref. (17) & & & & & & & & & & 3.75 & & \\
\hline Ref. (18) & & & & & & & & & & 5.95 & & \\
\hline Ref. (19) & & & & & & & & & & & 142 & 158 \\
\hline
\end{tabular}

i) Ratio of Sommerfeld coefficient $\mathrm{Y}$ of the FM state and that of the AFM state (YFM/YAFM),

ii) Bulk modulus of the AFM state $\left(B_{\mathrm{AFM}}\right)$, iii) Bulk modulus of the FM state $\left(B_{\mathrm{FM}}\right)$ 
The band structures of the AFM and FM states are investigated and are shown in Figs. 6(a)-(c), respectively. The two band structures are considerably different. In particular, in the FM state, the number of branches crossing the Fermi surface in the minority spin state is higher than that in the majority spin state. In the AFM state, a hole pocket appears at the center of the $\Gamma$ point. (a)

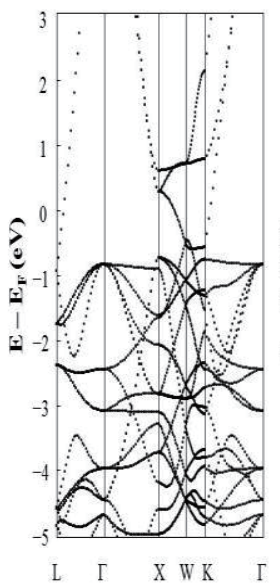

(b)

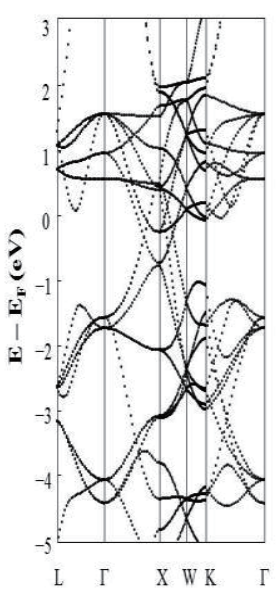

(c)

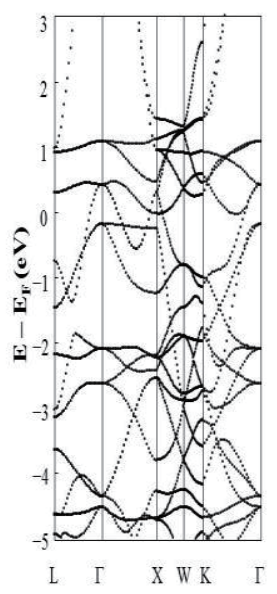

Fig. 6 Obtained band structures of (a) the FM state for the majority spin state, (b) the FM state for the minority spin state, and (c) the AFM state.

\section{Conclusion}

In summary, the electronic and magnetic structures of $\mathrm{FeRh}$ are investigated by using first principles calculations including appropriately chosen values of $U$ for the Fe3d and $\mathrm{Rh}_{4 \mathrm{~d}}$ electrons, and we succeed in explaining the reported AFM-FM phase transition experiments for the first time by obtaining $\Delta E$ consistent with the experimental reports, also for the first time. Moreover, other obtained physical quantities are also consistent with the experimental reports. We will attempt to clarify the mechanism behind the AFM-FM phase transition by using the knowledge obtained in the present analysis.

Acknowledgements The authors are indebted to colleagues on our team for advising us on computer system support.

\section{References}

1) K. G. Sanderman: Scripta Mater., 67, 566 (2012).

2) A. Fujita, S. Fujieda, Y. Hasegawa, and K. Fukamichi: Phys. Rev. B, 67, 104416 (2003).

3) N. H. Dung, Z. Q. Ou, L. Caron, L. Zhang, D. T. C. Thanh, G. A. de Wijs, R. A. de Groot, K. H. J. Buschow, and E. Brück: Adv. Energy Mater., 1, 1215 (2011).

4) M. P. Annaorazov, K. A. Asatryan, G. Myalikgulyev, S. A. Nikitin, A. M. Tishin, and A. L. Tyurin: Cryogenics, 32, 867 (1992).

5) L. Caron, X. F. Miao, J. C. P. Klaasse, S. Gama, and E. Brück: Appl. Phys. Lett., 103, 112404 (2013).

6) A. M. Tishin and Y. I. Spichkin: Int. J. Refrig., 37, 223 (2014).

7) M. Fallot: Ann. Phys., 10, 291 (1938).
8) A. I. Zakharov, A. M. Kadomtseva, R. Z. Levitin, and E. G. Ponyatovskii: Sov. Phys. JETP, 19, 1348 (1964).

9) T. Miyanaga, T. Itoga, T. Okazaki, and K. Nitta: J. Phys. Conf. Ser., 190, 012097 (2009).

10) D. W. Cooke, F. Hellman, C. Baldasseroni, C. Bordel, S. Moyerman, and E. E. Fullerton: Phys. Rev. Lett., 109, 255901 (2012).

11) G. Shirane, R. Nathans, and C. W. Chen: Phys. Rev., 134, A1547 (1964).

12) J. B. McKinnon, D. Melville, and E. W. Lee: J. Phys. C, 3 , S46 (1970).

13) B. K. Ponomarev: Sov. Phys, JETP, 36, 105 (1973).

14) T. Zhou, M. K. Cher, L. Shen, J. F. Hu, and Z. M. Yuan: Phys. Lett. A, 377, 3052 (2013).

15) J.-S. Lee, E. Vescovo, L. Plucinski, C. M. Schneider, and C.-C. Kao: Phys. Rev. B, 82, 224410 (2010).

16) A. X. Gray, D. W. Cooke, P. Krüger, C. Bordel, A. M. Kaiser, S. Moyerman, E. E. Fullerton, S. Ueda, Y. Yamashita, A. Gloskovskii, C. M. Schneider, W. Drube, K. Kobayashi, F. Hellman, and C. S. Fadley: Phys. Rev. Lett., 108, 257208 (2012).

17) P. Tu, A. J. Heeger, J. S. Kouvel, and J. B. Comly: J. Appl. Phys., 40, 1368 (1969).

18) J. Ivarsson, G.R. Pickett, and J. Tóth: Phys. Lett. A, 35, 167 (1971).

19) J. A. Ricodeau and D. Melville: J. Phys. F, 2, 337 (1972).

20) C. Kittel: Phys. Rev., 120, 335 (1960).

21) V. L. Moruzzi and P. M. Marcus: Phys. Rev. B, 46, 2864 (1992).

22) V. L. Moruzzi and P. M. Marcus: Solid State Commun., 83, 735 (1992).

23) A. Szajek and J. A. Morkowski: Physica B, 193, 81 (1994).

24) M. E. Gruner, E. Hoffmann, and P. Entel: Phys. Rev. B, 67, 064415 (2003).

25) R. Y. Gu and V. P. Antropov: Phys. Rev. B, 72, 012403 (2005).

26) L. M. Sandratskii and P. Mavropoulos: Phys. Rev. B, 83, 174408 (2011).

27) Y. Kaneta, S. Ishino, Y. Chen, S. Iwata, and A. Iwase: Jpn. J. Appl. Phys., 50, 105803 (2011).

28) A. Deák, E. Simon, L. Balogh, L. Szunyogh, M. dos Santos Dias, and J. B. Staunton: Phys. Rev. B, 89, 224401 (2014).

29) G. Kresse and J. Furthmüller: Comput. Mat. Sci., 6, 15 (1996).

30) G. Kresse and J. Furthmüller: Phys. Rev. B, 54, 11169 (1996).

31) P. E. Blöchl: Phys. Rev. B, 50, 17953 (1994).

32) G. Kresse and D. Joubert: Phys. Rev. B, 59, 1758 (1999).

33) J. P. Perdew, K. Burke, and M. Ernzerhof: Phys. Rev. Lett., 77, 3865 (1996).

34) V. I. Anisimov, J. Zaanen, and O.K. Andersen: Phys. Rev. B, 44, 943 (1991)

35) S. L. Dudarev, G. A. Botton, S. Y. Savrasov, C. J. Humphreys, and A. P. Sutton: Phys. Rev. B, 57, 1505 (1998).

36) M. Methfessel and A. T. Paxton: Phys. Rev. B, 40, 3616 (1989).

37) H. J. Monkhorst and J. D. Pack: Phys. Rev. B, 13, 5188 (1976).

38) $\Delta E$ obtained with $E_{\text {cut }} 830 \mathrm{eV}$ and an $11 \times 11 \times 11$ mesh is compared with that obtained with $E_{\text {cut }} 680 \mathrm{eV}$ and an $11 \times 11 \times 11$ mesh. The difference in $\Delta E$ for the two conditions is less than $0.01 \mathrm{meV} . \Delta E$ obtained with $E_{\text {cut }} 830 \mathrm{eV}$ and an $11 \times 11 \times 11$ mesh is compared with that obtained with $E_{\text {cut }}$ $830 \mathrm{eV}$ and a $15 \times 15 \times 15$ mesh. The difference in $\Delta E$ for the two conditions is about $0.1 \mathrm{meV}$.

Received Mar. 04, 2016; Accepted Apr. 23, 2016 